\title{
Superoxide dismutase and ascorbate peroxidase genes in Antarctic endemic brown alga Ascoseira mirabilis (Ascoseirales, Phaeophyceae): data mining of a de novo transcriptome
}

https://doi.org/10.1515/bot-2020-0025

Received April 23, 2020; accepted October 9, 2020; published online October 21, 2020

\begin{abstract}
The Antarctic endemic brown alga Ascoseira mirabilis is physically and physiologically well adapted to the extreme polar environment. To better understand the molecular strategies associated with stress adaptation, the transcriptome of $A$. mirabilis was sequenced, and its antioxidant enzyme genes were identified and compared with other algae. A total of 126,576 unigenes with a mean length of $734 \mathrm{bp}$ and N50 of $1174 \mathrm{bp}$ was assembled from the transcriptome data. Phylogenetic analysis revealed that dramatic evolutionary changes had occurred in the brown algal superoxide dismutases (SODs) and class I peroxidases that were not consistent with the phylogeny of the species. Fe/Mn SODs are more prevalent in brown algae than in red and green algae. Two additional Fe-SODs in A. mirabilis were phylogenetically closely related to those of green algae, but not to those in other brown algae. We also identified three $A$. mirabilis ascorbate peroxidase (APx) genes that had a different origin from other brown algal APxs. The SOD and APx genes specifically identified in $A$. mirabilis will be crucial for understanding the evolution of the algal antioxidant enzymes that contribute to ecological success under extreme environmental conditions in the Antarctic region.
\end{abstract}

Keywords: Antarctic brown alga; ascorbate peroxidase; Ascoseira mirabilis; superoxide dismutase; transcriptomic analysis.

\footnotetext{
*Corresponding author: Chenlin Liu, Key Lab of Marine EcologicalEnvironmental Science and Technology, Ministry of Natural Resources, Qingdao 266061, China; and Laboratory for Marine Biology and Biotechnology, Qingdao National Laboratory for Marine Science and Technology, Qingdao 266000, China, E-mail: ch.lliu@163.com

Xiuliang Wang, Laboratory for Marine Biology and Biotechnology, Qingdao National Laboratory for Marine Science and Technology, Qingdao 266000, China; and Institute of Oceanology, Chinese Academy of Sciences, Qingdao 266071, China
}

\section{Introduction}

The brown alga Ascoseira mirabilis Skottsberg 1907 is endemic to the Antarctic region (Wiencke et al. 2007). The thallus of $A$. mirabilis is diploid and monecious. It has a typical Fucales-like life history, with isogamous sexual reproduction (Clayton 1987). A. mirabilis is the only species in the Ascoseirales, and it diverged from other brown algae during the Cretaceous Period ( 100 Ma; Kawai et al. 2015). As one of the dominant species of seaweed communities in the Antarctic Peninsula, the life strategy, ecophysiology and ecology of $A$. mirabilis have been investigated since the 1980s (Clayton 1987; Clayton and Ashburner 1990; Gómez and Wiencke 1998; Huovinen and Gómez 2013; Mystikou et al. 2014; Roleda et al. 2007).

Ascoseira. mirabilis shows annual acclimation to nearfreezing and freezing temperatures and winter darkness (Wiencke et al. 2009). It is a shade adapted species and can grow under very low light, even under the sea ice, due to its high photosynthetic efficiency and low light requirements for photosynthesis (Gómez et al. 1995). Its photosynthetic rates are highest in September, with an optimal temperature range of $1-10{ }^{\circ} \mathrm{C}$ (Gómez et al. 1995). A. mirabilis also shows high UV tolerance with high concentrations of phlorotannin and other antioxidants (Huovinen and Gómez 2013; Núñez-Pons et al. 2018). Efficient photosynthetic recovery and DNA repair mechanisms were also observed in the gametes of $A$. mirabilis when UV-B stress was removed (Roleda et al. 2007). These characteristics represent considerable acclimation strategies of $A$. mirabilis to cope with the extreme polar environment (Gómez and Huovinen 2015). However, little has been reported on the molecular mechanism related to the adaptation of A. mirabilis to the harsh Antarctic environment.

Antioxidant enzymes play important roles in the acclimation of macroalgae to extreme temperature and UV-irradiation (Cruces et al. 2017; Wiencke and Bischof 2012). Studies have shown that the gene expression levels and enzyme scavenger activities of reactive oxygen species 
(ROS) are up-regulated when the macroalgae are exposed to abiotic stresses such as UV irradiance and temperature, indicating that these ROS scavengers can enhance tolerance to various stresses. There was an increase in superoxide dismutase (SOD) and ascorbate peroxidase (APx) enzyme activities under light stress in the brown alga Lessonia spicata (Cruces et al. 2017). Transcripts encoding an APx in Saccharina latissima were significantly upregulated under low temperatures and high light (Heinrich et al. 2012). In Arctic macroalgae, fast acclimation to changes in the environmental radiation conditions are supported by enhanced ROS-scavenging systems (Aguilera et al. 2002). UV radiation and enhanced temperature also induce the antioxidant activities of Antarctic brown algae (Cruces et al. 2013). Desmarestia anceps from the upper subtidal can tolerate high UV-B irradiance and are characterized by high activities of SOD. In contrast, individuals at lower subtidal positions show decreased SOD activities and a decreased UV tolerance for photosynthesis, suggesting that the antioxidative potential of SODs plays a crucial role in UV tolerance in endemic Antarctic brown macroalgae (Rautenberger et al. 2013). The transcriptomic analysis of $D$. anceps revealed that high and constitutive expression of chloroplastic ROS-scavenging enzymes was observed in response to abiotic changes in temperature and $\mathrm{CO}_{2}$ (Iñiguez et al. 2017).

The constitutions of antioxidant enzyme genes in different algal taxa showed variety (Rezayian et al. 2019). But studies on the evolutionary relationships of algal antioxidant enzymes are sparse, especially on the brown algal genes (Maruta et al. 2016; Miller 2012; Zámocký et al. 2014). Considering the harsh environment of Antarctica, the capacity of $A$. mirabilis to acclimate to low temperatures, seasonal light fluctuations, and solar UV radiation is an important determinant of its biological fitness (Wiencke et al. 2007). To better understand the molecular strategies associated with the environmental adaptations of $A$. mirabilis, the transcriptome of $A$. mirabilis was analyzed, and genes in the antioxidant enzyme family were examined. The transcriptomic data will provide useful information for understanding the adaptation strategy of brown algae to extreme environmental conditions.

\section{Materials and methods}

\subsection{Algae collection}

Ascoseira. mirabilis was collected in the intertidal zone near Changcheng Station, King George Island, South Shetland Islands $\left(62.216^{\circ} \mathrm{S}\right.$, $58.960^{\circ} \mathrm{W}$ ) in February 2018 during the 34th Chinese National
Antarctic Research Exploration. Healthy thalli were picked and washed several times with sterile seawater. Then the samples were stored in a $-150{ }^{\circ} \mathrm{C}$ refrigerator on the Xiangyanghong 01 vessel until RNA extraction was performed.

\subsection{Total RNA extraction and RNA-Seq}

The algal sample was immersed in liquid nitrogen and ground to a fine powder. Total RNA was extracted using an RNAprep pure plant kit (Tiangen, Beijing, China) according to the manufacturer's instructions. The concentration, quality, and integrity of RNA were determined using a NanoDrop spectrophotometer (Thermo Scientific). The sequencing library was generated using the TruSeq RNA Sample Preparation Kit (Illumina, San Diego, CA, USA). DNA fragments with ligated adapter molecules on both ends were selectively enriched using the Illumina PCR Primer Cocktail in a 15-cycle PCR and purified using the AMPure XP system (Beckman Coulter, Beverly, CA, USA). Products were quantified using the Agilent high sensitivity DNA assay on a Bioanalyzer 2100 system (Agilent). The library was then sequenced by Shanghai Personal Biotechnology Cp. Ltd on a Hiseq platform (Illumina), generating $150 \mathrm{bp}$ paired-end sequences.

\subsection{Transcriptome assembly and functional annotation}

The original data in FASTQ format (Raw Data) were filtered using Cutadapt (v1.15) software (Martin 2011) to obtain high-quality sequences. Pair-end reads with primer or adapter sequences were removed. Reads with more than $10 \%$ of all bases below the Q20 quality threshold or with more than $5 \%$ of all bases labeled unknown nucleotides (Ns) were filtered from the total reads. We used Trinity (v2.5.1) software to assemble clean reads into transcripts (Grabherr et al. 2011). The longest transcript of each gene was extracted as the representative sequence of the gene, called a unigene. The databases used for unigene functional annotation included the NR (NCBI non-redundant protein sequences, http://www.ncbi.nlm.nih.gov, E-value $\left.<10^{-5}\right)$, Swiss-Prot (https://web.expasy.org/, E-value $<10^{-5}$ ), GO (Gene Ontology, http://www.geneontology.org/), and EggNOG (evolutionary genealogy of genes: non-supervised orthologous groups, http://eggnog.embl.de/version_3.0/) databases. Pathway analysis was performed using the Kyoto Encyclopedia of Genes and Genomes (KEGG) annotation service KAAS using the Bi-directional Best Hit (BBH) method (Moriya et al. 2007) with an E value cutoff of $10^{-5}$.

\subsection{Sequence alignment and phylogenetic analysis}

The sequences from A. mirabilis involved in the phylogenetic analysis are shown in Supplementary Table S1. Protein localizations were predicted with Predotar (https://urgi.versailles.inra.fr/ predotar/). Predotar recognizes the $\mathrm{N}$-terminal targeting sequences of classically targeted precursor proteins (Small et al. 2004). The peroxidase family sequences were classified with PeroxiScan (http://peroxibase.toulouse.inra.fr/) (Fawal et al. 2013). Multiple sequence alignment of protein sequences was performed with the ClustalW program (Thompson et al. 2002) implemented in the DNAstar package (DNAStar, Inc.) using default parameters. Phylogenetic analysis was performed using MEGA X software (Kumar et al. 2018). Phylogenetic trees of protein sequences were constructed using the Neighbor-Joining (NJ) method. The reliability 
of the obtained trees was tested using a bootstrapping method with 1000 replicates.

\section{Results and discussion}

\subsection{Assembly and annotation of transcriptome sequences}

A photo of $A$. mirabilis is shown in Figure 1. And the taxonomy was confirmed with the $18 \mathrm{~S}$ rRNA gene (MT573535).

A total of 46,006,350 raw reads were obtained from the RNA-seq data of $A$. mirabilis. The raw data were deposited in the Short Read Archive (SRA) database of NCBI (https://www.ncbi.nlm.nih.gov/) with a BioProject ID of PRJNA529774, and a BioSample accession number of SAMN11283831. After adapters, ambiguous reads, and lowquality reads were filtered out, we retained approximately $45,715,494$ clean reads $(6.87 \mathrm{~GB})$. A total 126,576 unigenes were assembled with a mean length of $734 \mathrm{bp}$ and N50 of 1174 bp. There were 17,262 unigenes longer than the N50 sequence, which benefitted the unigene annotation and analysis. The average GC content of the A. mirabilis unigenes was $48.60 \%$, which is close to the GC content of the brown algal species Sargassum vulgare $(50.31 \%$; Kumar et al. 2017).

Within the 126,576 unigenes, only 12,945 and 11,386 unigenes, respectively, matched deposited sequences in the NR and SwissProt database. A search against the EggNOG database showed that a total of 11,554 unigenes were classified into 25 categories. Except for "General function prediction only" and "Function unknown", most of the transcripts were assigned to "Posttranslational modification, protein turnover, chaperones” (1343),

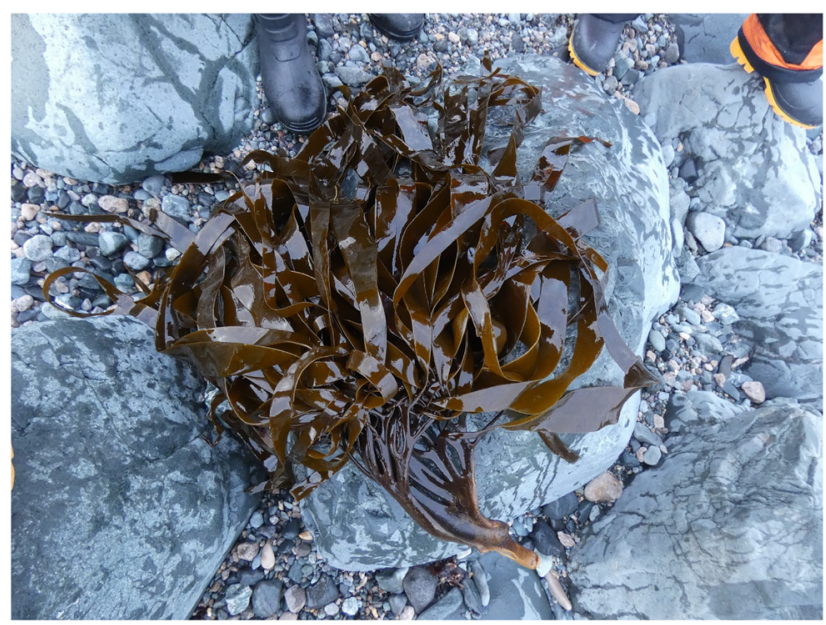

Figure 1: Morphology of Ascoseira mirabilis.
"Signal transduction mechanisms" (962), "Translation, ribosomal structure and biogenesis" (803), "Replication, recombination and repair" (681) and "Carbohydrate transport and metabolism” (549; Figure 2). A total of 4150 unigenes were assigned to at least one Gene Ontology (GO) term and divided into 44 functional groups. The GO terms "metabolic process" (2018) and "cellular process" (2119) were predominant in the biological process category, the terms "cell" (1806) and "cell part" (1789) were predominant in the cellular component category, and the terms "catalytic activity" (2025) and "binding” (1748) were predominant in the molecular function category. The predominant GO annotation categories in the A. mirabilis transcriptome were consistent with those in Saccharina japonica (Liang et al. 2014). Functional annotation and classification by alignment against the KEGG database showed that 3277 unigenes of $A$. mirabilis were mapped onto the metabolic pathways. The numbers of assembled and annotated unigenes in A. mirabilis were similar to those of other brown algae, such as S. japonica (Wang et al. 2013) and S. vulgare (Kumar et al. 2017).

To date, transcriptomic data from approximately 30 species of brown algae have been submitted to GenBank, and several brown algal genomes have been sequenced. Currently, however, most of our knowledge on the function of brown algal proteins is based on studies in model plants, animals, yeast, or bacteria. The Phaeophyceae evolved independently and experienced a large genome expansion during their divergence from plants over the past one billion years (Yoon et al. 2004). Because of insufficient evidence of protein similarities to plants and other algae, most of the expanded families in brown algae were classified as proteins with unknown function. This is partly due

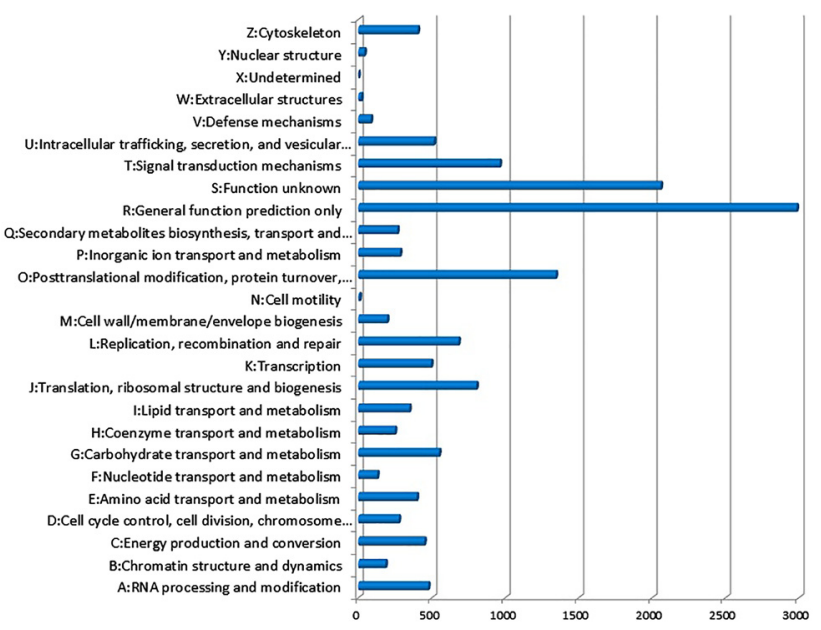

Figure 2: Evolutionary genealogy of genes: non-supervised orthologous groups (EggNOG) annotation of the Ascoseira mirabilis transcriptome. 
to the limited amount of brown algal transcriptomic sequence annotations (Dittami et al. 2020; Ye et al. 2015).

\subsection{Superoxide dismutase (SOD) gene family in $A$. mirabilis}

Transcriptomic sequencing data obtained from A. mirabilis, and publicly available genomic data from several other algal species, were used to identify SOD genes to provide insights into the evolution and distribution of SODs. In the transcriptome of $A$. mirabilis, seven $\mathrm{Fe} / \mathrm{Mn}$-SODs and one CuZn-SOD gene were identified by searching the protein family database (Pfam) (PF00081, PF00080). Six Fe/MnSOD and two CuZn-SOD genes in the genome of Ectocarpus siliculosus (Cock et al. 2010), two Fe/Mn-SOD and two CuZn-SODs in the red alga Chondrus crispus genome (Collén et al. 2013), two Fe/Mn-SOD and two CuZn-SODs in the red alga Gracilariopsis chorda genome (Lee et al. 2018), and six $\mathrm{Fe} / \mathrm{Mn}$-SODs in the green alga Chlamydomonas reinhardtii genome (https://phytozome.jgi.doe.gov/) were detected. Seven Fe/Mn-SOD and two CuZn-SOD genes were found in the transcriptome of the brown alga Sargassum thunbergii (Liu et al. 2014). Our results indicated that the SOD gene family was expanded in brown algae, compared to red algae and green algae.

A phylogenetic tree based on translated amino acid sequences of SOD genes from eukaryotic algae, bacteria, cyanobacteria, and plants was constructed (Figure 3). $\mathrm{Fe} / \mathrm{Mn}-\mathrm{SOD}$ sequences in all of these species were clustered into one group with high bootstrap value support. MnSODs and Fe-SODs are generally accepted to have arisen from a common ancestor from a gene duplication event (Fink and Scandalios 2002; Wolfe-Simon et al. 2005). The $\mathrm{Fe} / \mathrm{Mn}$-SOD cluster in our tree was divided into three moderately supported sister groups, Fe-, $\mathrm{Mn}$-, and Fe/MnSOD, according to the naming mode of SOD isoforms in C. reinhardtii (https://phytozome.jgi.doe.gov/). Mn-SOD was distributed among red algae, brown algae, green algae, and bacteria. Four genes of $A$. mirabilis belonged to the Mn-SOD family, divided into four separate branches. All of them except TRINITY DN36738 cO g1 matched homologs in other brown algae. TRINITY DN36738 c0 g1 was a partial sequence and its exact metalloform is uncertain. TRINITY DN49611 cO g1 clustered with the red algal MnSODs and a homolog in S. thunbergii but not in E. siliculosus. These brown algal isoforms in the red algal branch might be inherited from secondary endosymbiotic processes from the Rhodophyta. TRINITY DN30304 c2 g2 clustered with the Mn- SOD sequence from bacteria and might have originated from bacteria via horizontal gene transfer. TRINITY DN29750 c5 g1 clustered with Mn-SODs from green algae and plants and might have been inherited from another organelle acquisition process. This phenomenon indicated brown algal Mn-SOD genes have multiple origins and are much more diversified than the Mn-SOD genes of red and green algae.

The Fe-SOD subgroup contained sequences from brown algae, green algae, plants, bacteria, and cyanobacteria, but not from red algae. Functional Fe-SOD may

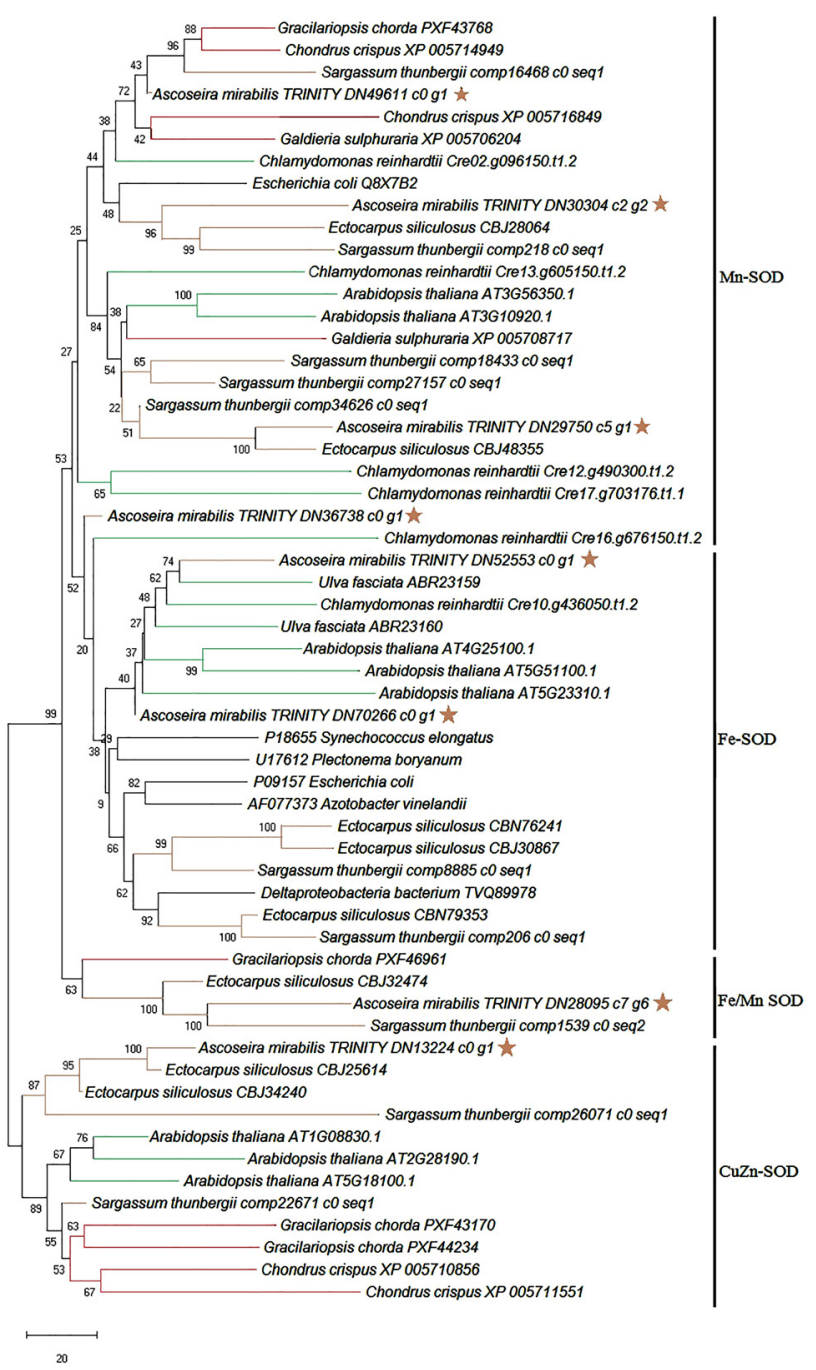

Figure 3: Neighbor-joining phylogenetic tree based on the translated amino acids of superoxide dismutase (SOD) genes from brown, red and green algae, with bootstrap values indicated at the nodes. All of the identified SOD genes that encoded amino acid sequences in the transcriptomes of Ascoseira mirabilis and Sargassum thunbergii, and in the genomes of Ectocarpus siliculosus, Chondrus crispus, Gracilariopsis chorda and Chlamydomonas reinhardtii are shown in the tree. The colors of the branches reflect different taxa: brown = brown algae; red = red algae; green = green algae and plants; black = bacteria and cyanobacteria. SODs from $A$. mirabilis are marked with brown stars. The separate groups are marked with vertical lines on the right. 
have been lost during evolution, leading to their absence in Rhodophytes (Matsuzaki et al. 2004). Two isoforms of A. mirabilis were phylogenetically related to the Fe-SODs in green algae and plants, and had no homologs from other brown algae. Unlike the Fe-SODs in A. mirabilis, the FeSODs in E. siliculosus and $S$. thunbergii were clustered with prokaryotic SODs. This result suggests different evolutionary origins of Fe-SODs among species of brown algae. It was believed that $\mathrm{Mn} / \mathrm{FeSODs}$ are more prevalent in more primitive prokaryotes and eukaryotes and reflect very early events in their evolution (Miller 2012). We speculate that the Fe-SODs in A. mirabilis were acquired by gene transfer from an endosymbiont, while the brown algae $E$. siliculosus and $S$. thunbergii might have acquired the Fe-SOD genes from bacteria by horizontal gene transfer. Different origins of Fe-SODs between A. mirabilis and other brown algae might correlate with their ecological environmental adaptations. The third Fe/Mn-SOD subgroup was specifically present in brown algae and the red alga G. chorda, but was absent in other red algal species, such as $C$. crispus. Their specific metalloforms were unclear, and no homologs were found in other organisms.

Based on our phylogenetic tree (Figure 3), CuZn-SOD isoforms were distributed in both brown algae and red algae but were absent in green algae, as previously reported (Pilon et al. 2011). CuZn-SODs from brown and red algae were clustered into two separated groups, except for one CuZn-SOD from $S$. thunbergii, that is closely related to red algal and plant SODs, and distantly related to other brown algal SODs (Figure 3). It is believed that CuZn-SOD genes in eukaryotic algae were obtained by gene transfer to the host cell from the genome of an organelle donor, perhaps independently of organelle acquisition (Miller 2012; Wolfe-Simon et al. 2005). The distribution of brown algal CuZn-SODs also suggests their separate evolutionary origins.

Previous phylogenetic studies on algal SODs were mainly focused on Chlorophytes, Rhodophytes, Cyanobacteria and diatoms, and few brown algal SODs were considered (Miller 2012; Wolfe-Simon et al. 2005). Our analysis indicates that the evolution of the SOD gene family is not exactly correlated with the evolution of the organism, such as the specific Fe/Mn-SOD gene in G. chorda and the CuZn-SOD gene in S. thunbergii and the Fe-SODs in A. mirabilis. The presence of these SOD enzymes may be related to the alga's habitat niche. The unique SOD genes in A. mirabilis appear to be essential to its evolutionary success in adapting to the harsh extremes of the Antarctic environment, and deserved further investigation.

\subsection{Class I heme peroxidase genes in A. mirabilis}

Ascorbate peroxidases (APx), cytochrome c peroxidases ( $\mathrm{CcP}$ ) and catalase-peroxidases (KatG) are Class I heme peroxidases. Based on NR and Pfam (PF00141) annotation, five peroxidase genes were identified in the $A$. mirabilis transcriptome. After analysis with PeroxiScan, the genes were predicted to be three APx and two CcP genes. We also found three APxs and two CcPs in the published genome of E. siliculosus (Cock et al. 2010). This is consistent with the records in PeroxiBase (Fawal et al. 2013). PeroxiBase currently records peroxidase sequences from 25 brown algal species with available genomic or transcriptomic data. Of the 25 brown algae in the database, 10 species lack class I peroxidase genes, six species possess two CcP genes, and only E. siliculosus has three APx and two CcP genes (http://peroxibase.toulouse.inra.fr/organism/get listing_org/16). The brown alga $S$. thunbergii was absent in PeroxiBase, and two APx genes were found in its transcriptome (Liu et al. 2014).

Together with the translated APx amino acid sequences from red algae, diatoms, green algae and plants, an APx and CcPs phylogenetic tree of brown algae was constructed (Figure 4). Except for one APx of A. mirabilis that clustered in the red algal group, the APxs and CcPs of brown algae were roughly divided into four groups. Based on the classification of $A$. mirabilis sequences, brown algal groups I and IV were APx branches and groups II and III were CcP branches. TRINITY DN29997 c5 g1 and TRINITY $D N 30935 \mathrm{c} 2 \mathrm{~g} 2$ are CcP encoding genes in A. mirabilis, with each having a close relative in E. siliculosus. Red algal CcP was unidentified in our study according to the Pfam (PF00141) annotation and phylogenetic analysis.

Both brown and red algal groups were phylogenetically separated from green algae, diatoms, and plants based on the APx sequences. The divergence of red algal groups from the groups of plants and other algae has been proven by Teixeira et al. (2004) and Zámocký et al. (2014). The only molecular evolution analysis performed on brown algal APxs was for an APx gene from E. siliculosus, which was classified into the chloroplastic group, and clustered with unicellular green algae, diatoms and red algae in a phylogenetic tree constituted with plant and algal APxs (Maruta et al. 2016). However, based on our analysis, brown algal APxs are actually phylogenetically separated from APxs of green algae and plants.

Although A. mirabilis and E. siliculosus had an identical number of APx genes, only one isoform (TRINITY DN27945 c4 g2) in A. mirabilis was phylogenetically close to 


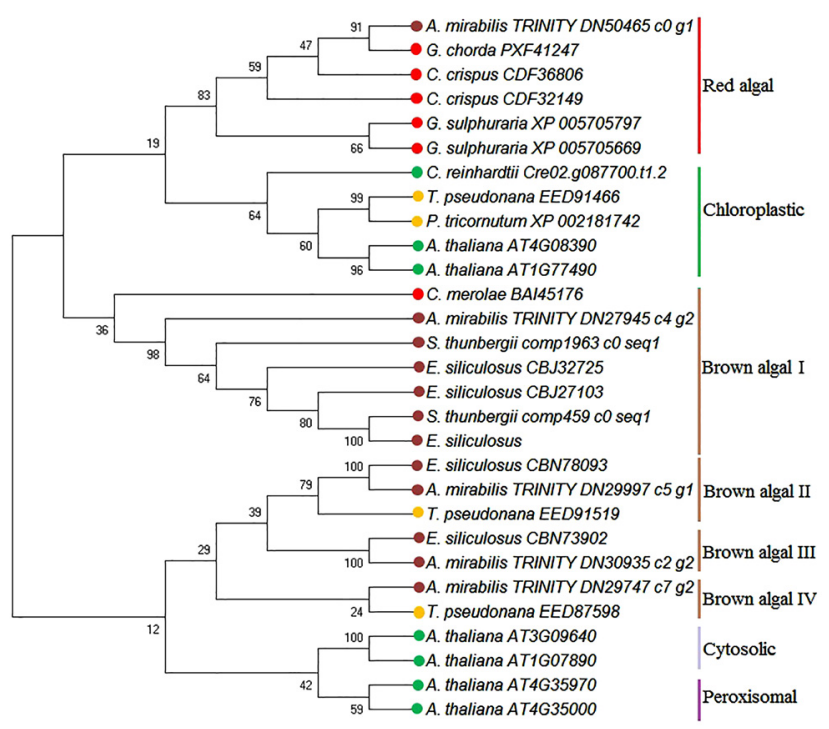

Figure 4: Neighbor- joining bootstrap consensus phylogenetic tree based on the translated amino acids of ascorbate peroxidases (APx) from algae and plants, with bootstrap values indicated at the nodes. The tree shows all of the APxs in the brown algae Ascoseira mirabilis, Sargassum thunbergii and Ectocarpus siliculosus (brown dots), the red algae Chondrus crispus, Gracilariopsis chorda, Galdieria sulphuraria and Cyanidioschyzon merolae strain 10D (red dots), the diatoms Phaeodactylum tricornutum CCAP 1055/1 and Thalassiosira pseudonana CCMP1335 (yellow dots), the green alga Chlamydomonas reinhardtii and the plant Arabidopsis thaliana (green dots). The separate groups are marked with vertical lines.

the three APxs in E. siliculosus and the two APxs in S. thunbergii. TRINITY DN50465 cO g1was clustered with the red algal APxs, and TRINITY DN29747 $c 7 \mathrm{~g} 2$ had a weak conjunction with a diatom APx (Figure 4). This result indicates that the origin and evolution of APxs in A. mirabilis are more complex than for the APxs in other brown algae, which might relate to its environmental adaptation. Multiple sequence alignments with APxs in A. mirabilis,
E. siliculosus and red algae demonstrated both high conservation of the active site residues and some variability (Figure 5). All of the APxs in brown and red algae possessed essential residues for heme binding. TRINITY DN27945 c4 g2 and APxs of E. siliculosus in brown algal group I had two chloroplastic insertion domains, similar to typical chloroplastic APxs in higher plants (Maruta et al. 2016). In TRINITY DN50465 CO g1 and other red algal type APxs, the chloroplastic domains were absent, as previously reported by Teixeira et al. (2004). Two predicted chloroplastic domains were found in TRINITY_DN29747_c7_g2 from brown algal group IV, but the protein localization prediction of Predotar (Small et al. 2004), indicated that this APx was likely located in the endoplasmic reticulum (ER; 0.66), and not in the plastid (0.09). Since the original site of peroxisomes is the ER (Tabak et al. 2008), we suggest that TRINITY_DN29747_c7_g2 might be a peroxisomal APx. This result, if true, is very important, since the peroxisomal APXs have previously been found only in the Streptophytes of eukaryotic algae (Zámocký et al. 2014).

We also found a catalase-peroxidase (KatG) gene in the A. mirabilis transcriptome. Of 25 brown algae in PeroxiBase, only two species possess KatG genes, with four genes in E. siliculosus (although we found only three KatGs in its genome) and one gene in Sargassum henslowianum. Phylogenetic analysis indicated that brown algal KatGs evolution was not consistent with the phylogeny of the species (Figure 6). They were more closely related to KatGs from bacteria than to KatGs from other eukaryotic organisms. In the phylogenetic tree of the peroxidase-catalase superfamily sequences of Zámocký et al. (2014), KatG evolved into two branches, a major KatG clade including sequences from all bacterial phyla and fungal KatG genes. Furthermore, there was a putative minor clade of KatGs from eukaryotic photosynthetic algae and heterotrophic Stramenopiles. Our results indicate that, unlike KatGs in

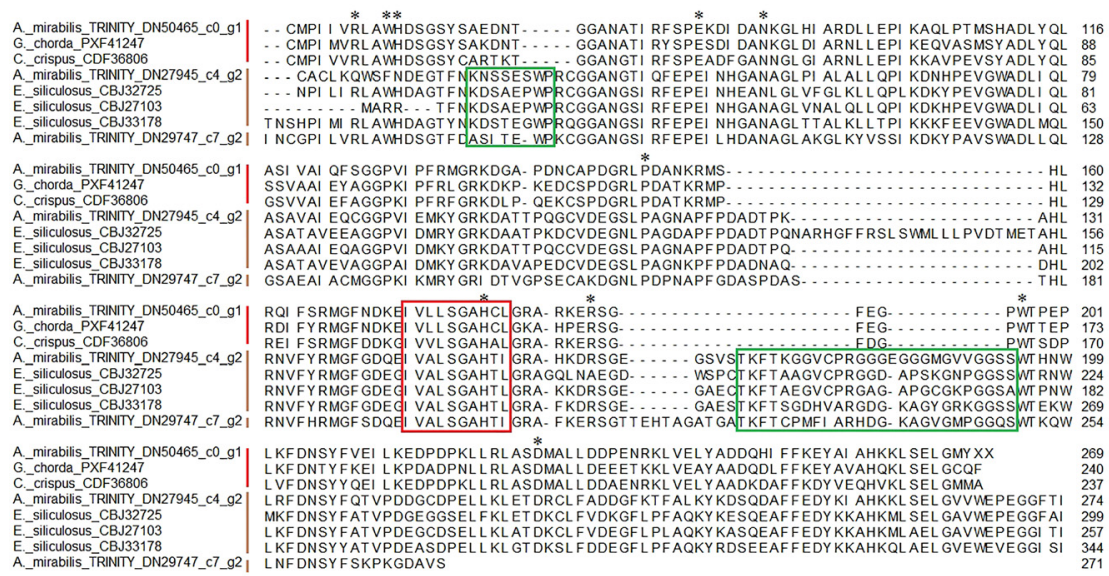

Figure 5: Multiple sequence alignment of predicted ascorbate peroxidase (APX) and Cytochrome $c$ peroxidase (CCP) amino acid sequences from Ascoseira mirabilis, Ectocarpus siliculosus, Chondrus crispus and Gracilariopsis chorda. The separate groups are marked with vertical lines. Essential residues involved in catalysis are labeled with asterisks. Two extra chloroplastic domains in APXs in the chloroplastic group (green boxes), and a heme-binding site (red box) are shown following Teixeira et al. (2004). 


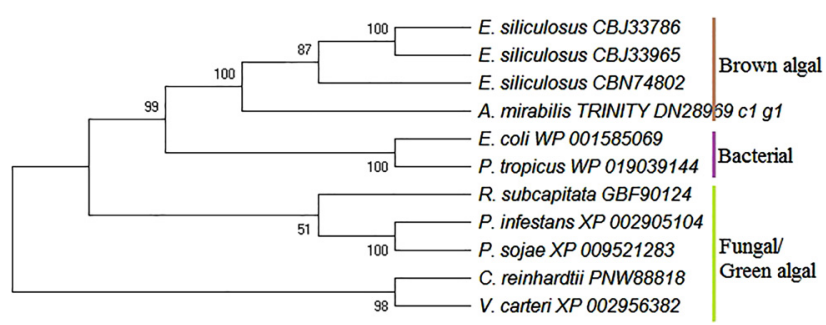

Figure 6: Neighbor-joining bootstrap consensus phylogenetic tree based on the translated amino acids of catalase-peroxidase (KatG) sequences from algae, fungi and bacteria, with bootstrap values indicated at the nodes. The brown algal KatG was inherited from bacteria by horizontal gene transfer. The KatG sequences in the brown algae Ascoseira mirabilis and Ectocarpus siliculosus, the bacteria Escherichia coli and Psychroflexus tropicus, the fungi Phytophthora infestans T30-4, Phytophthora sojae and Raphidocelis subcapitata, and the green algae Chlamydomonas reinhardtii and Volvox carteri $f$. nagariensis are shown.

other eukaryotic photosynthetic algae, the evolution of KatGs in brown algae was probably more primitive than that in fungi, and some species of brown algae might acquire KatGs from bacteria by horizontal gene transfer. Algal peroxidases originated and evolved from both vertical descent and horizontal gene transfer as observed in several clades within the evolutionary history of this superfamily (Zamocky et al. 2010).

\section{Conclusions}

With the development of high throughout sequencing techniques, more and more transcriptomic and genomic data from brown algae were produced. However, due to the evolutionary divergence from other organisms, only a limited number of brown algal gene sequences have been annotated and discussed. We chose a narrow but important examination of the species-specific molecular evolution of antioxidant enzyme genes in A. mirabilis and other eukaryotic algae, that was undetected in previous studies. Our results indicate that the evolution of the antioxidant gene family is not exactly correlated with the evolution of the organism. Dramatic evolutionary changes have occurred in brown algal SODs and class I peroxidases, which are more complex than those in other eukaryotic photosynthetic algae. Brown algal SOD genes have multiple origins and are far more diversified than those of red and green algae. The origin and evolution of APxs in A. mirabilis are more complex than the APxs in other brown algae. Considering its important ecological status, the physiology of the Antarctic brown alga A. mirabilis growing in extreme environments has been studied extensively; however, the molecular adaptation mechanisms have not been sufficiently investigated. The expanded SOD and APX gene families in A. mirabilis appear to be crucial for understanding the evolution of algal antioxidant enzymes that contribute to the ecological adaptation. Further characterization of the gene expression and biochemical analyses of these expanded genes under abiotic stress conditions might contribute to our understanding of the evolution and structure-function relationships of antioxidant enzyme genes in brown algae.

Acknowledgments: We wish to thank Professor John van der Meer for the English writing modification in this manuscript.

Author contribution: All the authors have accepted responsibility for the entire content of this submitted manuscript and approved submission.

Research funding: This work was supported by the 34th Chinese National Antarctic Research Exploration with the Xiangyanghong 01 scientific expedition vessel (JDKC0518013), and a grant from the National Key R\&D programme of China (2018YFC1406705).

Conflict of interest statement: The authors declare no conflicts of interest regarding this article.

\section{References}

Aguilera, J., Dummermuth, A., Karsten, U., Schriek, R., and Wiencke, C. (2002). Enzymatic defences against photooxidative stress induced by ultraviolet radiation in Arctic marine macroalgae. Polar Biol. 25: 432-441.

Clayton, M.N. (1987). Isogamy and a fucalean type of life history in the Antarctic brown alga Ascoseira mirabilis (Ascoseirales, Phaeophyta). Bot. Mar. 30: 447-506.

Clayton, M.N., and Ashburner, C.M. (1990). The anatomy and ultrastructure of "conducting channels" in Ascoseira mirabilis (Ascoseirales, Phaeophyceae). Bot. Mar. 33: 63-70.

Cock, J.M., Sterck, L., Rouzé, P., Scornet, D., Allen, A.E., Amoutzias, G., Anthouard, V., Artiguenave, F., Aury, J.M., Badger, J.H., et al. (2010). The Ectocarpus genome and the independent evolution of multicellularity in brown algae. Nature 465: 617-621.

Collén, J., Porcel, B., Carré, W., Ball, S.G., Chaparro, C., Tonon, T., Barbeyron, T., Michel, G., Noel, B., Valentin, K., et al. (2013). Genome structure and metabolic features in the red seaweed Chondrus crispus shed light on evolution of the Archaeplastida. Proc. Natl. Acad. Sci. U.S.A. 110: 5247-5252.

Cruces, E., Huovinen, P., and Gómez, I. (2013). Interactive effects of UV radiation and enhanced temperature on photosynthesis, phlorotannin induction and antioxidant activities of two subAntarctic brown algae. Mar. Biol. 160: 1-13.

Cruces, E., Rautenberger, R., Rojas-Lillo, Y., Cubillos, V.M., ArancibiaMiranda, N., Ramírez-Kushel, E., and Gómez, I. (2017). 
Physiological acclimation of Lessonia spicata to diurnal changing PAR and UV radiation: differential regulation among down-regulation of photochemistry, ROS scavenging activity and phlorotannins as major photoprotective mechanisms. Photosynth. Res. 131: 145-157.

Dittami, S.M., Corre, E., Brillet-Guéguen, L., Lipinska, A.P., Pontoizeau, N., Aite, M., Avia, K., Caron, C., Cho, C.H., Collen, J., et al. (2020). The genome of Ectocarpus subulatus - a highly stress-tolerant brown alga. Mar. Genom.: 100740, https://doi. org/10.1016/j.margen.2020.100740.

Fawal, N., Li, Q., Savelli, B., Brette, M., Passaia, G., Fabre, M., Mathé, C., and Dunand, C. (2013). PeroxiBase: a database for large-scale evolutionary analysis of peroxidases. Nucleic Acids Res. 41: D441-D444.

Fink, R.C. and Scandalios, J.G. (2002). Molecular evolution and structurefunction relationships of the superoxide dismutase gene families in angiosperms and their relationship to other eukaryotic and prokaryotic superoxide dismutases. Arch. Biochem. Biophys. 399: 19-36.

Gómez, I. and Huovinen, P. (2015). Lack of physiological depth patterns in conspecifics of endemic antarctic brown algae: $a$ trade-off between UV stress tolerance and shade adaptation. PloS One 10: e0134440.

Gómez, I. and Wiencke, C. (1998). Seasonal changes in C, N, and major organic compounds and their significance to morphofunctional processes in the endemic Antarctic brown alga Ascoseira mirabilis. Polar Biol. 19: 115-124.

Gómez, I., Wiencke, C., and Weykam, G. (1995). Seasonal photosynthetic characteristics of Ascoseira mirabilis (Ascoseirales, Phaeophyceae) from King George Island, Antarctica. Mar. Biol. 123: 167-172.

Grabherr, M.G., Haas, B.J., Yassour, M., Levin, J.Z., Thompson, D.A., Amit, I., Adiconis, X., Fan, L., Raychowdhury, R., Zeng, Q., et al. (2011). Full-length transcriptome assembly from RNA-seq data without a reference genome. Nat. Biotechnol. 29: 644-652.

Heinrich, S., Valentin, K., Frickenhaus, S., John, U., and Wiencke, C. (2012). Transcriptomic analysis of acclimation to temperature and light stress in Saccharina latissima (Phaeophyceae). PloS One 7: e44342.

Huovinen, P., and Gómez, I. (2013). Photosynthetic characteristics and UV stress tolerance of Antarctic seaweeds along the depth gradient. Polar Biol. 36: 1319-1332.

Iñiguez, C., Heinrich, S., Harms, L., and Gordillo, F.J.L. (2017). Increased temperature and $\mathrm{CO} 2$ alleviate photoinhibition in Desmarestia anceps: from transcriptomics to carbon utilization. J. Exp. Bot. 68: 3971-3984.

Kawai, H., Hanyuda, T., Draisma, S.G.A., Wilce, R.T., and Andersen, R.A. (2015). Molecular phylogeny of two unusual brown algae, Phaeostrophion irregulare and Platysiphon glacialis, proposal of the Stschapoviales ord. nov. and Platysiphonaceae fam. nov. and a re-examination of divergence times for brown algae orders. J. Phycol. 51: 918-928.

Kumar, A., Castellano, I., Patti, F.P., Delledonne, M., and Buia, M.C. (2017). Molecular response of sargassum vulgare to acidification at volcanic $\mathrm{CO}_{2}$ vents - insights from de novo transcriptomic analysis. Mol. Ecol. 26: 2276-2290.

Kumar, S., Stecher, G., Li, M., Knyaz, C., and Tamura, K. (2018). MEGA $X$ : molecular evolutionary genetics analysis across computing platforms. Mol. Biol. Evol. 35: 1547-1549.
Lee, J., Yang, E.C., Graf, L., Yang, J.H., Qiu, H., Zelzion, U., Chan, C.X., Stephens, T.G., Weber, A.P., Boo, G.H., et al. (2018). Analysis of the draft genome of the red seaweed Gracilariopsis chorda provides insights into genome size evolution in Rhodophyta. Mol. Biol. Evol. 35: 1869-1886.

Liang, X., Wang, X., Chi, S., Wu, S., Sun, J., Liu, C., Chen, S., Yu, J., and Liu, T. (2014). Analysis of Saccharina japonica transcriptome using the high-throughput DNA sequencing technique and its vanadium-dependent haloperoxidase gene. Acta Oceanol. Sin. 33: $27-36$.

Liu, F., Sun, X., Wang, W., Liang, Z., and Wang, F. (2014). De novo transcriptome analysis-gained insights into physiological and metabolic characteristics of Sargassum thunbergii (Fucales, Phaeophyceae). J. Appl. Phycol. 26: 1519-1526.

Maruta, T., Sawa, Y., Shigeoka, S., and Ishikawa, T. (2016). Diversity and evolution of ascorbate peroxidase functions in chloroplasts: more than just a classical antioxidant enzyme?. Plant Cell Physiol. 57: 1377-1386.

Martin, M. (2011). Cutadapt removes adapter sequences from highthroughput sequencing reads. EMBnet J. 17: 10-12.

Matsuzaki, M., Misumi, O., Shin, I.T., Maruyama, S., Takahara, M., Miyagishima, S.Y., Mori, T., Nishida, K., Yagisawa, F., Yoshida, Y., et al. (2004). Genome sequence of the ultrasmall unicellular red alga Cyanidioschyzon merolae 10D. Nature 428: 653-657.

Miller, A.F. (2012). Superoxide dismutases: ancient enzymes and new insights. FEBS Letters 586: 585-595.

Moriya, Y., Itoh, M., Okuda, S., Yoshizawa, A.C., and Kanehisa, M. (2007). KAAS: an automatic genome annotation and pathway reconstruction server. Nucleic Acids Res. 35: W182-185.

Mystikou, A., Peters, A.F., Asensi, A.O., Fletcher, K.I., Brickle, P., van West, P., Convey, P., and Küpper, F.C. (2014). Seaweed biodiversity in the south-western Antarctic Peninsula: surveying macroalgal community composition in the Adelaide Island/ Marguerite Bay region over a 35-year time span. Polar Biol. 37: 1607-1619.

Núñez-Pons, L., Avila, C., Romano, G., Verde, C., and Giordano, D. (2018). UV-protective compounds in marine organisms from the Southern Ocean. Mar. Drugs 16: 336.

Pilon, M., Ravet, K., and Tapken, W. (2011). The biogenesis and physiological function of chloroplast superoxide dismutases. Biochim. Biophys. Acta 1807: 989-998.

Rautenberger, R., Wiencke, C., and Bischof, K. (2013). Acclimation to UV radiation and antioxidative defence in the endemic Antarctic brown macroalga Desmarestia anceps along a depth gradient. Polar Biol. 36: 1779-1789.

Rezayian, M., Niknam, V., and Ebrahimzadeh, H. (2019). Oxidative damage and antioxidative system in algae. Toxicol. Rep. 6: 1309-1313.

Roleda, M.Y., Zacher, K., Wulff, A., Hanelt, D., and Wiencke, C. (2007). Photosynthetic performance, DNA damage and repair in gametes of the endemic Antarctic brown alga Ascoseira mirabilis exposed to ultraviolet radiation. Austral Ecol. 32: 917-926.

Small, I., Peeters, N., Legeai, F., and Lurin, C. (2004). Predotar: a tool for rapidly screening proteomes for $\mathrm{N}$-terminal targeting sequences. Proteomics 4: 1581-1590.

Tabak, H.F., van der Zand, A., and Braakman, I. (2008). Peroxisomes: minted by the ER. Curr. Opin. Cell Biol. 20: 393-400.

Teixeira, F.K., Menezes-Benavente, L., Rogério, M., and Márcia, M.P. (2004). Analysis of the molecular evolutionary history of the 
ascorbate peroxidase gene family: inferences from the rice genome. J. Mol. Evol. 59: 761-770.

Thompson, J.D, Gibson, T.J., and Higgins, D.G. (2002). Multiple sequence alignment using ClustalW and ClustalX. Curr. Protoc. Bioinf. 1: 2-3.

Wang, W.J., Wang, F.J., Sun, X.T., Liu, F.L., and Liang, Z.R. (2013). Comparison of transcriptome under red and blue light culture of Saccharina japonica (phaeophyceae). Planta 237: 1123-1133.

Wiencke, C., and Bischof, K. (2012). Seaweed responses to environmental stress: reactive oxygen and antioxidative strategies. Seaweed Biol. 219: 109-132.

Wiencke, C., Clayton, M.N., Gómez, I., Iken, K., Lüder, U.H., Amsler, C.D., Karsten, U., Hanelt, D., Bischof, K., and Dunton, K. (2007). Life strategy, ecophysiology and ecology of seaweeds in polar waters. Rev. Environ. Sci. Biotechnol. 6: 95-126.

Wiencke, C., Gómez, I., and Dunton, K. (2009). Phenology and seasonal physiological performance of polar seaweeds. Bot. Mar. 52: 585-592.

Wolfe-Simon, F., Grzebyk, D., Schofield, O., and Falkowski, P.G. (2005). The role and evolution of superoxide dismutases in algae. J. Phycol. 41: 453-465.

Ye, N., Zhang, X., Miao, M., Fan, X., Zheng, Y., Xu, D., Wang, J., Zhou, L., Wang, D., Gao, Y., et al. (2015). Saccharina genomes provide novel insight into kelp biology. Nat. Commun. 6: 6986.

Yoon, H.S., Hackett, J.D., Ciniglia, C., Pinto, G., and Bhattacharya, D. (2004). A molecular timeline for the origin of photosynthetic eukaryotes. Mol. Biol. Evol. 21: 809-818.

Zámocký, M., Furtmüller, P.G., and Obinger, C. (2010). Evolution of structure and function of Class I peroxidases. Arch. Biochem. Biophys. 500: 45-57.

Zámocký, M., Gasselhuber, B., Furtmüller, P.G., and Obinger, C. (2014). Turning points in the evolution of peroxidase-catalase superfamily: molecular phylogeny of hybrid heme peroxidases. Cell. Mol. Life Sci. 71: 4681-4696.

Supplementary Material: The online version of this article offers supplementary material (https://doi.org/10.1515/bot-2020-0025).

\section{Bionotes}

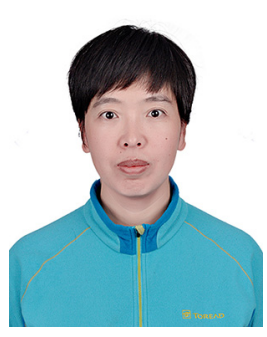

\author{
Chenlin Liu \\ Key Lab of Marine Ecological-Environmental \\ Science and Technology, Ministry of Natural \\ Resources, Qingdao 266061, China \\ Laboratory for Marine Biology and \\ Biotechnology, Qingdao National Laboratory \\ for Marine Science and Technology, Qingdao \\ 266000, China \\ ch.lliu@163.com
}

Chenlin Liu is a professor at the Key Lab of Marine EcologicalEnvironmental Science and Technology, MNR. Her research topic is acclimation strategies of algae in extreme polar environments. She uses transcriptomic and comparative algal genomic methods to explore the genes that play important roles in the acclimation of algae to extreme temperatures and UV-irradiation. She also works on the impact of climatic and anthropogenic factors on the biodiversity and community constitution of polar algae.

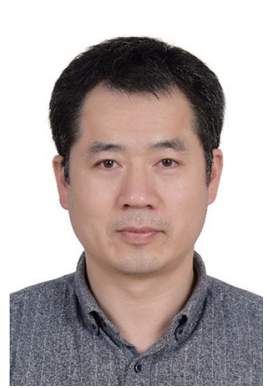

\section{Xiuliang Wang}

Laboratory for Marine Biology and Biotechnology, Qingdao National Laboratory for Marine Science and Technology, Qingdao 266000, China

and Institute of Oceanology, Chinese Academy of Sciences, Qingdao 266071, China

Xiuliang Wang is a senior researcher at the Experimental Marine Biology Laboratory, Institute of Oceanology, Chinese Academy of Sciences. He has studied and published on Saccharina japonica germplasm and its quantitative traits. He is also interested in the study of evolution of brown seaweeds under the circumstances of climatic change and anthropogenic influences. 